\title{
PREDICTIVE CONTROL FOR TRAJECTORY TRACKING AND DECENTRALIZED NAVIGATION OF MULTI-AGENT FORMATIONS
}

\author{
IONELA PRODAN *,**, SORIn OLARU* , Cristina STOICA* , SilviU-IUlian NiCUlESCU ** \\ * Automatic Control Department \\ SUPELEC Systems Sciences (E3S), 3 Joliot Curie, 91192, Gif sur Yvette, France \\ e-mail: \{ionela.prodan, sorin.olaru, cristina.stoica\}@supelec.fr \\ ${ }^{* *}$ Laboratory of Signals and Systems \\ SUPELEC-CNRS, 3 Joliot Curie, 91192, Gif sur Yvette, France \\ e-mail: \{ionela.prodan, silviu.niculescu\}@lss.supelec.fr
}

\begin{abstract}
This paper addresses a predictive control strategy for a particular class of multi-agent formations with a time-varying topology. The goal is to guarantee tracking capabilities with respect to a reference trajectory which is pre-specified for an agent designed as the leader. Then, the remaining agents, designed as followers, track the position and orientation of the leader. In real-time, a predictive control strategy enhanced with the potential field methodology is used in order to derive a feedback control action based only on local information within the group of agents. The main concern is that the interconnections between the agents are time-varying, affecting the neighborhood around each agent. The proposed method exhibits effective performance validated through some illustrative examples.
\end{abstract}

Keywords: multi-agent systems, linear systems, model predictive control, potential function, polyhedral function.

\section{Introduction}

Control and coordination of multi-agent systems, such as pedestrians in a crowd, vehicles, spacecraft and unmanned vehicles, are emerging as a challenging field of research. The advances in network design, information, control synthesis and sensors technology allow nowadays large groups of agents to be coordinated and controlled in an effective manner for various tasks in evaluating the safety of social infrastructures (Helbing et al., 2000; Fang et al., 2010), efficient flow of traffic (Van den Berg et al., 2004; Baskar et al., 2006), water control for irrigation canals, water supply and sewer networks (Overloop et al., 2010; Negenborn et al., 2009) and deep space observation (Mesbahi and Hadaegh, 2001; Massion et al., 2008). In addition, there exist several classes of multi-agent systems where the interconnections between the agents could be time-varying (e.g., traffic control, pedestrian behavior, etc.). Guaranteeing stability with the existing cooperative control techniques is still an open problem for multi-agent systems with time-varying (constrained) topologies. This paper addresses a new methodology based on predictive control in order to address some of these difficulties; illustrative examples prove the proposed methodology interesting.

Collision avoidance is often the most difficult problem in the context of managing multiple agents, since certain (static or dynamic) constraints are non-convex. A common point of most publications dealing with the collision avoidance problem is the hypothesis of pointwise agents, which is far from the conditions in real world applications. In many of them the relative positioning between agents becomes important, such as large interferometer construction from multiple telescopes or air traffic management (two aircraft are not allowed to approach each other closer than a specific alert distance).

A key idea for the treatment of collision avoidance problems is represented by Mixed-Integer Programming (MIP) (Osiadacz et al., 1990; Richards and How, 2002; Bemporad and Morari, 1999), which has the ability to include non-convex constraints and discrete decisions in the optimization problem. However, despite its modeling capabilities and the availability of good solvers, MIP has serious numerical drawbacks. As stated by Garey and 
Johnson (1979), mixed-integer techniques are NP-hard, i.e., the computational complexity increases exponentially with the number of binary variables used in the problem formulation. A method for reducing the number of binary variables is detailed by Stoican et al. (2011) with an application to the obstacle avoidance problem. Yet, the fundamental limitation of MIP complexity remains redoubtable.

A different class of methods for collision avoidance problems uses artificial potential fields (Khatib, 1986) to directly obtain feedback control actions steering the agents over the entire workspace. One shortcoming of this approach is the possible generation of traps (local minima). Relevant research on generating the so-called navigation functions that are free from local minima is available in the literature (Rimon and Koditschek, 1992). However, generating a navigation function is computationally involved and thus not suitable for many navigation problems.

There is a large literature dedicated to the formation control for collections of vehicles using the potential field approach. Jadbabaie et al. (2003) and Tanner et al. (2007) investigate the motions of vehicles modeled as double integrators. Their objective is for the vehicles to achieve a common velocity while avoiding collisions with obstacles and/or agents assumed to be points. The derived control laws involve graph Laplacians for an associated undirected graph and also nonlinear terms resulting from artificial potential functions. Roussos and Kyriakopoulos (2010) consider a decentralized navigation of multiple agents operating in a spherical workspace. Navigation functions are used to derive control laws for point-wise agents with an associated disk of a predefined radius around them.

In the present paper, we revise the preliminary results of Prodan et al. (2010; 2011) and introduce enhancements in the control design method which enables decentralized decision making for a leader/followers group of agents. The aim of this work is twofold:

- First, to provide a generic framework for non-point-wise shapes which may define obstacles and/or safety regions around an agent.

- Second, to offer a novel control strategy derived from a combination of a constrained receding horizon and potential field techniques for the trajectory tracking problem, applied to multi-agent systems with time-varying topologies.

To the best of the authors' knowledge, there does not exist a similar method in the open literature. The methods that we propose can be applied to various practical applications (e.g., motion control of wheeled mobile robots (Michałek et al., 2009), path following control of nonholonomic mobile manipulators (Mazur and
Szakiel, 2009), the control of a mobile offshore base viewed as a string of modules that have to be kept aligned (Girard et al., 2001)).

First, we introduce two different constructions which take into consideration the shape of the convex region associated with a safety region of an agent or an obstacle. The proposed constructions can be further used with the various potential or navigation functions existing in the literature in order to have a complete multi-agent system. Second, through the rest of the paper a leader/followers strategy for the trajectory tracking problem is proposed. The agents are required to follow a pre-specified trajectory while keeping a desired inter-agent formation in time. We consider polyhedral safety regions for the agents and obstacles. A specified trajectory is generated for the leader using the differential flatness formalism (Fliess et al., 1995). Differentially flat systems are well suited to problems requiring trajectory planning as they circumvents the complexity of differential equations formalism by transforming the model description in an algebraic form, more suitable for open-loop control design. The most important aspect of flatness in our context (predictive control) is that it reduces the problem of trajectory generation to finding a trajectory of the so-called flat output of the system through the resolution of a system of algebraic equations. Furthermore, for the followers, we propose a potential field method which aims to follow the group leader and respect the formation specifications. These are realized through the use of a receding horizon approach (Camacho and Bordons, 2004; Rossiter, 2003; Mayne et al., 2000), both for the leaders and followers.

This paper is organized as follows. Section 2 presents two constructions that take into account the shape of a convex region defining an obstacle and/or a safety region around an agent. Considering the dynamics of the agents, Section 3 presents the trajectory tracking problem for a leader/followers formation. A reference trajectory is generated for the leader and, using predictive control, the tracking error is minimized. For the followers, a potential function is embedded within Model Predictive Control (MPC) in order to achieve the group formation with a collision free behavior. Further on, Section 4 presents illustrative simulation results. Finally, several concluding remarks are drawn in Section 5

The following notation will be used throughout the paper. Given a vector $v \in \mathbb{R}^{n},\|v\|_{\infty}:=\max _{i=1, \ldots, n}\left|v_{i}\right|$ denotes the infinity norm of $v$. Minkowski's addition of two sets $\mathcal{X}$ and $\mathcal{Y}$ is defined as $\mathcal{X} \oplus \mathcal{Y}=\{x+y: x \in \mathcal{X}, y \in \mathcal{Y}\} . \quad$ The interior of a set $S, \operatorname{Int}(S)$ is the set of all interior points of $S$. The collection of all possible $n_{c}$ combinations of binary variables is written as $\{0,1\}^{n_{c}}=\left\{\left(b_{1}, \ldots, b_{n_{c}}\right): b_{i} \in\{0,1\}, \forall i=1, \ldots, n_{c}\right\}$. Denote by $\mathbb{B}_{p}^{n}=\left\{x \in \mathbb{R}^{n}:\|x\|_{p} \leq 1\right\}$ the unit ball 
of norm $p$, where $\|x\|_{p}$ is the $p$-norm of a vector $x$. We let $x_{k+1 \mid k}$ denote the value of $x$ at time instant $k+1$, predicted upon the information available at time $k \in \mathbb{N}$.

\section{Prerequisites}

For safety and obstacle avoidance problems the feasible region in the space of solutions is a non-convex set. Usually this region is considered the complement of a (union of) convex region(s) which describes an obstacle and/or a safety region. Due to their versatility and relative low computational complexity, the polyhedra are the instrument of choice in characterizing these regions.

Let us define a bounded convex set in its polyhedral approximation, a polytope $S \subset \mathbb{R}^{n}$ through the implicit half-space description:

$$
S=\left\{x \in \mathbb{R}^{n}: h_{a} x \leq k_{a}, a=1, \ldots, n_{h}\right\},
$$

with $h_{a} \in \mathbb{R}^{1 \times n}, k_{a} \in \mathbb{R}$ and $n_{h}$ being the number of half-spaces. We focus on the case where $k_{a}>0$, meaning that the origin is contained in the strict interior of the polytopic region, i.e., $0 \in \operatorname{Int}(S)$. $S$ in (1),

By definition, every supporting hyperplane for the set

$$
\mathcal{H}_{a}=\left\{x \in \mathbb{R}^{n}: h_{a} x=k_{a}, a=1, \ldots, n_{h}\right\},
$$

will partition the space into two disjoin 11 regions:

$$
\begin{aligned}
& \mathcal{R}^{+}\left(\mathcal{H}_{a}\right)=\left\{x \in \mathbb{R}^{n}: \quad h_{a} x \leq k_{a}\right\}, \\
& \mathcal{R}^{-}\left(\mathcal{H}_{a}\right)=\left\{x \in \mathbb{R}^{n}:-h_{a} x \leq-k_{a}\right\}
\end{aligned}
$$

where $\mathcal{R}_{a}^{+}$and $\mathcal{R}_{a}^{-}$denote in a simplified formulation the complementary regions associated to the $a$-th inequality of (1).

In the following, we are interested in measuring the relative position of an agent with respect to such a region. In other words, we require a function which measures if and when a given state is inside or outside the polyhedral set (1). The forthcoming constructions will be introduced in a repulsive potential function to take into account the shape of the convex region in terms of (1). The repulsive potential will be further used in a predictive control context in order to derive a control action such that the collision avoidance inside the formation is satisfied.

2.1. Polyhedral function. Consider the class of (symmetrical) piecewise linear functionals defined using the specific shape of a polyhedral set. The following definitions will be instrumental for the rest of the paper.

\footnotetext{
${ }^{1}$ The relative interiors of these regions do not intersect but their closures have as common boundary the affine subspace $\mathcal{H}_{a}$.
}

Definition 1. (Minkowski function (Blanchini, 1995)) Any bounded convex set $S$ induces a Minkowski function defined as

$$
\mu(x)=\inf \{\alpha \in \mathbb{R}, \alpha \geq 0: x \in \alpha S\} .
$$

Definition 2. (Polyhedral function (Blanchini, 1995)) A polyhedral function is the Minkowski function of the polyhedral bounded convex set $S$ defined in (1). This function has the following expression:

$$
\mu(x)=\|F x\|_{\infty},
$$

where $F \in \mathbb{R}^{n_{h} \times n}$ is a full column matrix with $F_{a}=$ $h_{a} / k_{a}, a=1, \ldots, n_{h}$.

In fact, any polytope can be defined in terms of the Minkowski function (5). Indeed, there always exists a full column matrix $F \in \mathbb{R}^{n_{h} \times n}$ such that the polytope $S$ in (1) is equivalently defined as

$$
S=\left\{x \in \mathbb{R}^{n}: \mu(x) \leq 1\right\},
$$

with $\mu(x)$ defined by (6). From the avoidance point of view, the Minkowski function (5) denotes the inclusion of a value $x$ to the given polytope (7) if $\mu(x) \in[0,1]$. Conversely, if $\mu(x)>1$, then $x$ is outside the polytope (7).

Remark 1. Note that, if $k_{a}<0$ in (1), the origin is not contained in the strict interior of the polytopic region, i.e., $0 \notin \operatorname{Int}(S)$, then the polyhedral function can be brought to the form (6) by imposing

$$
F_{a}=\frac{h_{a}\left(x-x_{s}\right)}{k_{a}-h_{a} x_{s}}, \quad a=1, \ldots, n_{h},
$$

with $x_{s} \in \mathbb{R}^{n}$ being the analytic center of the polytope (1).

Note that the polyhedral function (6) is piecewise affine and continuous. This means that each of the inequalities which compose its definition can provide the maximum, an explicit description of these regions being

$$
\begin{aligned}
X_{a}=\left\{x \in \mathbb{R}^{n}: \frac{h_{a}}{k_{a}} x>\frac{h_{b}}{k_{b}} x, \forall a\right. & \neq b, \\
a, b & \left.=1, \ldots, n_{h}\right\} .
\end{aligned}
$$

The entire space can thus be partitioned in a union of disjoint regions $X_{a}$ which are representing in fact cones with a common point at the origin (respectively, at $x_{s}$ for the general case evoked in Remark 1 .

Practically, the polyhedral function (5) can be represented in the form

$$
\mu(x)=F_{a} x, \quad \forall x \in X_{a}, \quad a=1, \ldots, n_{h},
$$

and the piecewise affine gradient is defined as

$$
\nabla \mu(x)=F_{a}, \quad \forall x \in X_{a}, a=1, \ldots, n_{h} .
$$


Remark 2. Strictly speaking, the generalized gradient (11) is multivalued (the Minkowski function induced by a polytope is not differentiable in the classical sense, it is rather differentiable almost everywhere). However, a univocal candidate can be selected for the computations and such an approach is used in the rest of the paper. We mention that, alternatively, the explicit use of a multivalued expression of the gradient would not bring computational difficulties as long as the range of variation is bounded and can be represented by the extreme values in practice.

2.2. Sum function. The polyhedral functions presented in the previous section represent the basis for the construction of "exclusion" functions (or penalty functions).

Consider again the polytope defined in (1), and a piecewise linear function (introduced by Camacho and Bordons (2004)):

$$
\psi(x)=\sum_{a=1}^{n_{h}}\left(h_{a} x-k_{a}+\left|h_{a} x-k_{a}\right|\right) .
$$

The function (12) is zero inside the convex region (11) and increases linearly in the exterior, as the distance to the boundary increases.

The definition (12) describes in fact a continuous piecewise affine function over a partition of the state-space. Over each of the polyhedral cells composing this partition, the absolute values of $\left|h_{a} x-k_{a}\right|$ are constant resulting in a fixed affine form for $\psi(x)$. In order to explicitly describe the regions composing the partition, several additional theoretical notions will be introduced.

Definition 3. (Hyperplane arrangements (Ziegler, 1995)) A collection of hyperplanes $\mathbb{H}=\left\{\mathcal{H}_{a}\right\}$ with $a=$ $1, \ldots, n_{h}$ partition the space as a union of disjoint cells defined as follows:

$$
\mathcal{A}(\mathbb{H})=\bigcup_{l=1, \ldots, \gamma\left(n_{h}\right)} \underbrace{\left(\bigcap_{a=1, \ldots, n_{h}} \mathcal{R}^{\sigma_{l}(a)}\left(\mathcal{H}_{a}\right)\right)}_{A_{l}},
$$

where $\sigma_{l} \in\{-,+\}^{n_{h}}$ denotes all feasible combinations of the regions (3) and (4) obtained for the hyperplanes in $\mathbb{H}$ and $\gamma\left(n_{h}\right)$ denotes the number of feasible cells.

Note that the number of regions in the hyperplane arrangement is usually much greater than the number of regions (9) associated with the polyhedral function (10).

Therefore, the piecewise affine function (12) can be alternatively described as

$$
\begin{aligned}
\psi(x)=2 & \sum_{\substack{a=1 \\
\sigma_{l}=\{+\}}}^{n_{h}}\left(h_{a} x-k_{a}\right), \\
& \forall x \in \mathcal{A}_{l}, l=1, \ldots, \gamma\left(n_{h}\right) .
\end{aligned}
$$

The piecewise affine gradient of (14) is defined as

$$
\begin{aligned}
\nabla \psi(x)=2 \sum_{\substack{a=1 \\
\sigma_{l}=\{+\}}}^{n_{h}} h_{a}^{T}, \\
\quad \forall x \in \mathcal{A}_{l}, \quad l=1, \ldots, \gamma\left(n_{h}\right) .
\end{aligned}
$$

2.3. Example of the construction of repulsive potential functions. In this subsection the previous theoretical tools will be integrated in order to describe two types of piecewise affine functions which measure the position of a state with respect to the boundary of a polyhedral set defined in (1).

In order to exemplify their influence in a collision avoidance problem, we propose several repulsive potential functions constructed through the use of the formulations (10) and (14). The potential functions take into account the shape of a convex region as in Fig. 1, which can define a safety region for an agent and/or an obstacle. For the given convex region, Figs. 2 and 3 illustrate the polyhedral function and the sum function defined according to (10) and (14), respectively.

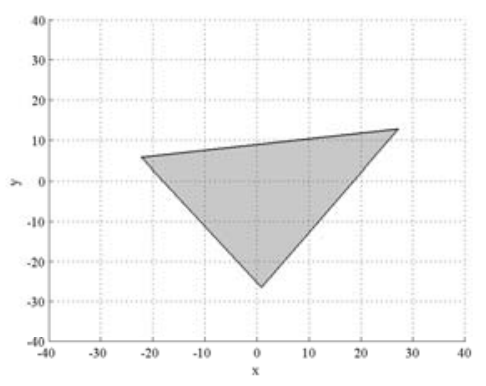

Fig. 1. Convex region.

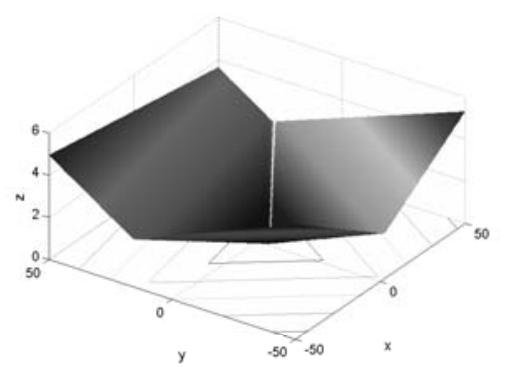

Fig. 2. Polyhedral function 10 of the convex region in Fig. 1

Furthermore, for control design, the construction based on the polyhedral function defined in (10) is proposed for the generation of a repulsive potential:

$$
V_{\mu}(\mu(x))=c_{1} e^{-\left(\mu(x)-c_{2}\right)^{2}},
$$




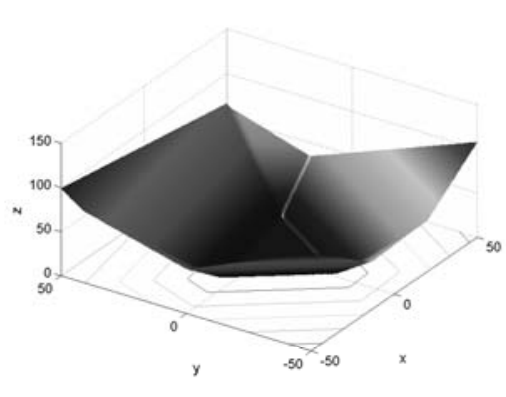

Fig. 3. Sum function (14) of the convex region in Fig. 1

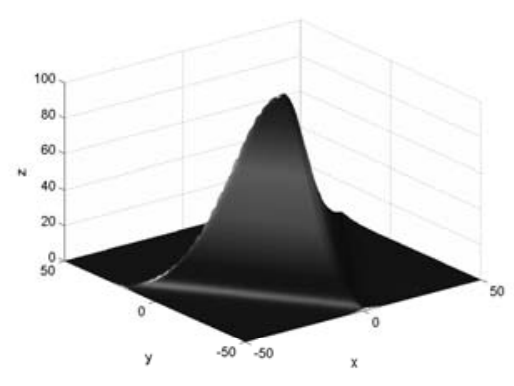

Fig. 4. Repulsive potential using the polyhedral function in Fig. 2

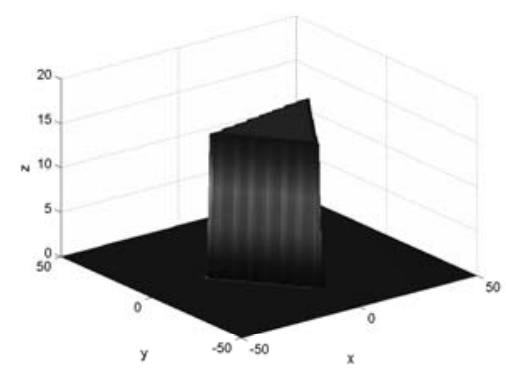

Fig. 5. Repulsive potential using the sum function in Fig. 3

where the parameters $c_{1}$ and $c_{2}$ are positive constants representing the strength and effect ranges of the repulsive potential.

An alternative repulsive potential using the sum function described in will be given by

$$
V_{\psi}(\psi(x))=\frac{c_{3}}{\left(c_{4}+\psi(x)\right)^{2}}
$$

with $c_{3}$ and $c_{4}$ being positive constants representing the strength and effect ranges of the repulsive potential (17).

Figures 4 and 5 illustrate the proposed functions (16) and 177, respectively, for the given convex region in Fig. 1] As can be seen, both the functions have a high value inside the polytopes and a low value outside them.
The repulsive potential will be further used in order to derive a control action such that the collision avoidance inside the formation is satisfied.

\section{Trajectory tracking for a leader/followers formation}

This section presents the formation trajectory tracking problem. The agents are required to follow a pre-specified trajectory while preserving a tight inter-agent formation in time. Each agent has an associated polyhedral safety region as defined in (1). Using a leader/followers approach, we generate a reference trajectory for the leader and formulate a receding horizon optimization problem in order to minimize the tracking error. For the followers, we propose a gradient method combined with a receding horizon approach which aims to follow the group leader and respect the collision avoidance formation specifications.

A set of $N_{a}$ linear systems (vehicles, pedestrians or agents in a general form) will be used to model the behavior of individual heterogeneous agents. The $i$-th system is described by the following continuous time dynamics:

$$
\dot{x}^{i}(t)=A_{c, i} x^{i}(t)+B_{c, i} u^{i}(t), \quad i=1, \ldots, N_{a},
$$

where $x^{i}(t) \in \mathbb{R}^{n}$ are the state variables and $u^{i}(t) \in$ $\mathbb{R}^{m}$ is the control input vector for the $i$-th agent. The components of the state are the position $p^{i}(t)$ and the velocity $v^{i}(t)$ of the $i$-th agent such that $x^{i}(t)=$ $\left[p^{i}(t) v^{i}(t)\right]^{T}$.

The problem of generating a reference trajectory for the leader (i.e., $i=l$ in (18)) is next summarized, along the line in the work of Van Nieuwstadt and Murray (1998).

3.1. Trajectory generation. The idea is to find a trajectory $\left(x^{l}(t), u^{l}(t)\right)$ that steers the model of the leader (18) with $i=l$ from an initial state $x_{0}$ to a final state $x_{f}$, over a fixed time interval $\left[t_{0}, t_{f}\right]$. Using flatness theory (Fliess et al., 1995; Van Nieuwstadt and Murray, 1998; Suryawan et al., 2010), the system is parameterized in terms of a finite set of variables $z^{l}(t)$ and a finite number of their derivatives:

$$
\begin{aligned}
& x^{l}(t)=\xi\left(z^{l}(t), \dot{z}^{l}(t), \ldots, z^{l,(q)}(t)\right), \\
& u^{l}(t)=\eta\left(z^{l}(t), \dot{z}^{l}(t), \ldots, z^{l,(q)}(t)\right),
\end{aligned}
$$

where

$$
z^{l}(t)=\Upsilon\left(x^{l}(t), u^{l}(t), \dot{u}^{l}(t), \ldots, u^{l,(q)}(t)\right)
$$

is called the flat output 2 . The generation of a reference trajectory will be based on the class of polynomial

\footnotetext{
${ }^{2}$ Hereafter, we assume that the characteristics necessary for flat trajectory (controllability and the existence of a flat output) are respected for the leader agent.
} 
functions. Using the parametrization (19) and imposing boundary constraints for the evolution of the differentially flat systems (De Doná et al., 2009), one can generate a reference trajectory $z_{\text {ref }}^{l}(t)$ by solving a system of linear equations. Therefore, the corresponding reference state and input for the system (18), with $i=l$, are obtained by replacing the reference flat output $z_{\text {ref }}^{l}(t)$, with $t \in\left[t_{0}, t_{f}\right]$ in 19):

$$
\begin{aligned}
& x_{\mathrm{ref}}^{l}(t)=\xi\left(z_{\mathrm{ref}}^{l}(t), \dot{z}_{\mathrm{ref}}^{l}(t), \ldots, z_{\mathrm{ref}}^{l,(q)}(t)\right), \\
& u_{\mathrm{ref}}^{l}(t)=\eta\left(\dot{z}_{\mathrm{ref}}^{l}(t), \ddot{z}_{\mathrm{ref}}^{l}(t), \ldots, z_{\mathrm{ref}}^{l,(q)}(t)\right),
\end{aligned}
$$

where $t \in\left[t_{0}, t_{f}\right]$.

In the rest of the paper, we use the discrete analog to the reference signals in 20. That is, a discrete-time model corresponding to Eqn. (18) is constructed upon a chosen sampling period $T_{s}$ by considering the time instants $t_{k}=k T_{s}$ :

$$
\begin{aligned}
x^{i}(k+1)=A_{i} x^{i}(k)+ & B_{i} u^{i}(k), \\
& k \in \mathbb{N}, \quad i=1, \ldots, N_{a},
\end{aligned}
$$

where $x^{i}(0)$ corresponds to the boundary condition in 20) and $u^{i}(k)=u^{i}\left(t_{k}\right)$. The pairs $\left(A_{i}, B_{i}\right)$ are given by

$$
A_{i}=e^{A_{c, i} T_{s}}, \quad B_{i}=\int_{0}^{T_{s}} e^{A_{c, i}\left(T_{s}-\theta\right)} B_{c, i} \mathrm{~d} \theta .
$$

Considering the discrete-time model of the leader (21) with $i=l$, we compare the measured state and input variables with the reference trajectory $\left(x_{\mathrm{ref}}^{l}(k), u_{\mathrm{ref}}^{l}(k)\right)$ which satisfies the nominal dynamics:

$$
x_{\text {ref }}^{l}(k+1)=A_{l} x_{\text {ref }}^{l}(k)+B_{l} u_{\text {ref }}^{l}(k) .
$$

Further on, the tracking error between the leader's state (21) and the state reference (22) becomes

$$
\tilde{x}^{l}(k+1)=A_{l} \tilde{x}^{l}(k)+B_{l} \tilde{u}^{l}(k),
$$

with $\tilde{u}^{l}(k)=u^{l}(k)-u_{\text {ref }}^{l}(k), \tilde{x}^{l}(k)=x^{l}(k)-x_{\text {ref }}^{l}(k)$.

Since the reference trajectory is available beforehand, an optimization problem which minimizes the tracking error for the leader can be formulated in a predictive control framework (Goodwin et al., 2006; Maciejowski, 2002). Consequently, the leader must follow the reference trajectory from the initial position to the desired one, using the available information over a finite time horizon in the presence of constraints 3 .

\footnotetext{
${ }^{3}$ The flat trajectory can also be generated to enforce obstacle avoidance at the trajectory planning stage. In this framework the obstacles can be modeled in terms of a convex safety region around each agent, as in (1). Even if the reference trajectory is generated over the entire interval $\left[t_{0}, t_{f}\right]$, intermediary points can be added along the trajectory in order to avoid obstacles on a specific time subinterval by redesigning the flat trajectory.
}

3.2. Predictive control for the leader. In what follows we present the predictive control problem, where optimization is performed to compute the control law for the leader. The discrete model of the leader (i.e., $i=l$ in (21) is used in a predictive control context which allows for the minimization of the tracking error.

A finite receding horizon implementation of the optimal control law is typically based on the real-time construction of a control sequence

$$
\tilde{\mathbf{u}}^{l}=\left\{\tilde{u}^{l}(k \mid k), \tilde{u}^{l}(k+1 \mid k), \cdots, \tilde{u}^{l}\left(k+N_{l}-1 \mid k\right)\right\}
$$

that minimizes the finite horizon quadratic objective function:

$$
\begin{aligned}
& \tilde{\mathbf{u}}^{*} \\
& =\arg \min _{\tilde{\mathbf{u}}^{l}}\left(\left\|\tilde{x}^{l}\left(k+N_{l} \mid k\right)\right\|_{P}\right. \\
& \left.\quad+\sum_{s=1}^{N_{l}-1}\left\|\tilde{x}^{l}(k+s \mid k)\right\|_{Q}+\sum_{s=0}^{N_{l}-1}\left\|\tilde{u}^{l}(k+s \mid k)\right\|_{R}\right),
\end{aligned}
$$

subject to

$$
\left\{\begin{array}{l}
\tilde{x}^{l}(k+s+1 \mid k)=A_{l} \tilde{x}^{l}(k+s \mid k)+B_{l} \tilde{u}^{l}(k+s \mid k), \\
\tilde{x}^{l}(k+s \mid k) \in X_{l}, \quad s=1, \ldots, N_{l}, \\
\tilde{u}^{l}(k+s \mid k) \in U_{l}, \quad s=1, \ldots, N_{l} .
\end{array}\right.
$$

Here $Q=Q^{T} \succeq 0, R \succ 0$ are positive definite weighting matrices, $P=P^{T} \succeq 0$ defines the terminal cost and $N_{l}$ denotes the prediction horizon for the leader. The optimization problem (24) has to be solved subject to the dynamical constraints (25). At the same time, other security or performance specifications can be added to the system trajectory. These physical limitations (velocity, energy or forces) are stated in terms of hard constraints on the internal state variables and input control action as in (25). Note that the sets $X_{l}$ and $U_{l}$ have to take into account the reference tracking type of the problem delineated in (24). Thus, the absolute limitations have to be adjusted according to the reference signals. In the original state space coordinates, these constraints will describe a tube around the reference trajectory. A finite horizon trajectory optimization is performed at each sample instant, the first component of the resulting control sequence being effectively applied. Then, the optimization procedure is reiterated using the available measurements based on the receding horizon principle (Camacho and Bordons, 2004).

\subsection{Decentralized predictive control for the follow-} ers. In this subsection, we present a control strategy which is a combination of MPC and the potential field control approach. The goal is to control the agents to achieve a formation while following the specified trajectory. The repulsive potential functions introduced in (16) and 17 produce a potential field. The negative 
gradient of this potential is the key element towards a collision free behavior for the agents. Globally, an attractive component of the potential function aims at maintaining a given formation. In this context, we provide a practical control design method which enables decentralized decision making for a leader/followers group of agents. The proposed method exhibits effective trajectory tracking performances while avoiding the centralized design which can be computationally demandingt.

Assume that each agent has an associated convex safety region as defined in (1). Before actually defining the potential function, let us recall a general result relative to convex sets which will be further used in the optimization problem.

Proposition 1. For any two convex sets $S_{1}, S_{2} \in \mathbb{R}^{n}$, the following relations are equivalent:

1. $S_{1} \cap S_{2}=\emptyset$,

2. $\{0\} \notin S_{1} \oplus\left\{-S_{2}\right\}$.

Proof. (Sketch) It suffices to note that if the origin is inside the set $S_{1} \oplus\left\{-S_{2}\right\}$, then, necessarily, there exist $x_{1} \in S_{1}$ and $x_{2} \in S_{2}$ such that $x_{1}-x_{2}=0$.

Corollary 1. Consider Agents $i$ and $j$ with the associated safety regions $S_{i}, S_{j}$ as defined in (1). Agent $i$ with the associated position $p^{i}$ does not intersect Agent $j$ with the position $p^{j}$ if an only if $p^{i} \notin S_{i j}\left(p^{j}\right)$, where

$$
S_{i j}\left(p^{j}\right) \triangleq\left\{p^{j}\right\} \oplus S_{j} \oplus\left\{-S_{i}\right\},
$$

with $i=1, \ldots, N_{a}, i \neq j$.

Remark 3. For the ease of the computation, the agents are considered unidimensional points in the position space. Even if they are characterized by a nonempty region $R_{i} \subset \mathbb{R}^{n}$, one can define the set in (1) as $\tilde{\mathcal{S}}_{i} \triangleq \mathcal{S}_{i} \oplus R_{i}$, where $R_{i}$ denotes the region describing the $i$-th agent.

Let us now assume the steering policy for each follower agent (i.e., $i \neq l$ in (21) ) based only on local state information from its nearest neighbors.

Definition 4. (Neighboring graph (Tanner et al., 2007)) An undirected graph $\mathcal{G}=\{\mathcal{V}, \mathcal{E}\}$ represents the nearest neighboring relations and consists of

- a set of vertices (nodes) $\mathcal{V}=\left\{n_{1}, n_{2}, \ldots, n_{N_{a}}\right\}$ indexed by the agents in the group;

\footnotetext{
${ }^{4}$ Besides computational expenses, the logistic difficulties can be mentioned: a centralized approach means that there exists a center which needs information from all the agents and has to send the control action to all agents. Such a construction may be difficult to implement and be prone to errors (e.g., the case of a radio linked system of agents and a non-neglected physical obstacle which cuts the communication inside a subgroup).
}

- a set of edges $\mathcal{E}=\left\{\left(n_{i}, n_{j}\right) \in \mathcal{V} \times \mathcal{V}: n_{i} \leftrightarrow n_{j}\right\}$, containing unordered pairs of nodes that represent neighboring relations.

The set of neighbors of Agent $i$ with $i=1, \ldots, N_{a}$ and $i \neq l$ can be defined as follows:

$$
\mathcal{N}_{i}(k) \triangleq\left\{j=1, \ldots, N_{a}:\left\|p^{i}(k)-p^{j}(k)\right\| \leq r, i \neq j\right\},
$$

where $r$ is the radius of the ball centered in $p^{i}$. Since the agents are in motion, their relative distances can change with time, affecting their neighboring sets (27). For each agent $i$, we define an inter-agent potential function which aims to accomplish the following objectives:

(i) collision avoidance between agents;

(ii) convergence to a group formation and following the leader.

To be specific, in our problem, the following inter-agent potential function is used:

$$
V_{i}\left(p^{i}, v^{i}\right)=\beta_{r} V_{i}^{r}\left(p^{i}\right)+\beta_{a} V_{i}^{a}\left(p^{i}, v^{i}\right), \quad \forall i \in \mathcal{N}_{i} .
$$

The two components of the potential function account for the objectives presented above and $\beta_{r}, \beta_{a}$ are weighting coefficients for each objective. For the $i$-th agent the total potential is formed by summing the potentials terms corresponding to each of its neighbors. Consequently, in our approach, the potential functions are designed as follows:

(i) $V_{i}^{r}\left(p^{i}\right)$ denotes the repulsive potentials that Agent $i$ sense from its neighbors:

$$
V_{i}^{r}\left(p^{i}\right)=\sum_{j \in \mathcal{N}_{i}} V_{i j}^{r}\left(p^{i}\right) .
$$

To implement this, the concepts introduced in Section 2.3, specifically the potential functions (16) or (17), are taken into account:

$$
V_{i j}^{r}\left(p^{i}\right)=\frac{c_{3}}{\left(c_{4}+\psi_{i j}\left(p^{i}\right)\right)^{2}}, \quad i \neq j, i \neq l,
$$

where $\psi_{i j}\left(p^{i}\right)$ is the sum function (14) induced by the polyhedral set defined in (26). Note that the repulsive component (30) takes into account the safety regions (26) associated with both the followers and the leader.

(ii) $V_{i}^{a}\left(p^{i}, v^{i}\right)$ denotes the attractive component between agents in order to achieve a formation and to follow the leader:

$$
V_{i}^{a}\left(p^{i}, v^{i}\right)=\sum_{j \in \mathcal{N}_{i}} V_{i j}^{a}\left(p^{i}, v^{i}\right)+\left\|p^{l}-p^{i}\right\|,
$$

for all $i \in \mathcal{N}_{i}$ and $i \neq l$. 
The second component denotes the relative distance between the leader and the followers. The first component $V_{i j}^{a}\left(x_{i}\right)$ has the following form:

$$
V_{i j}^{a}\left(p^{i}, v^{i}\right)=\log \left(\psi_{i j}^{2}\left(p^{i}\right)\right)+\beta_{v}\left(v^{i}-v^{j}\right),
$$

where $\beta_{v}$ denotes a weighting coefficient for which the agents velocities are synchronizing.

As can be observed in similar works based on potential function methods, the parameters of the potential field have to be tuned experimentally. It will be seen in the simulations that collision avoidance is realized for the chosen parameters. Also note that, for a potential function, a piecewise affine gradient can be computed using the results in Section 2 As in the works of Rimon and Koditschek (1992), as well as Tanner et al. (2007), the negative value of the gradient can be applied in order to derive a control action for Agent $i$. The direct approach has several shortcomings mentioned in Section 1.

In the following, we reformulate the optimization problem (24) for the followers, by using the potential-based cost function described in 28): Find a control sequence

$$
\mathbf{u}^{i}=\left\{u^{i}(k \mid k), u^{i}(k+1 \mid k), \cdots, u^{i}\left(k+N_{f}-1 \mid k\right)\right\}
$$

which minimizes the finite horizon nonlinear objective function:

$$
\mathbf{u}^{*}=\arg \min _{\mathbf{u}^{i}}\left(\sum_{s=0}^{N_{f}} V_{i}\left(p^{i}(k+s \mid k), v^{i}(k+s \mid k)\right) .\right.
$$

Here $N_{f}$ denotes the prediction horizon for the followers. In the optimization problem (33) we need to know the future values of the neighboring graph and the values of the state for the corresponding neighbors. All these elements are time-varying and difficult to estimate. For the ease of computation, we assume the following:

- The neighboring graph is considered to be constant along the prediction horizon, that is,

$$
\mathcal{N}_{i}(k+s \mid k) \triangleq \mathcal{N}_{i}(k)
$$

- The future values of the followers state are considered constant,

$$
x^{j}(k+s \mid k) \triangleq x^{j}(k) .
$$

- An estimate of the leader's state is provided by (23),

$$
x^{l}(k+s \mid k) \triangleq \tilde{x}_{\mathrm{ref}}^{l}(k+s) .
$$

Equations (34)-36 represent only a rough approximation of the future state of the agents.
Obviously, the MPC formulation can be improved by using prediction of the future state of the neighboring agents. Where feasible, this prediction may be provided by the agents themselves (Dunbar and Murray, 2006). Here a simplified approach was implemented for the followers (by assuming constant predictions) and using the reference trajectory for the leader.

Remark 4. The time-varying nature of the neighboring graph and the fact that the future values of the neighboring states and the leader state are unknown represent some of the computational limitations of the presented scheme. Moreover, the resulting cost function is nonlinear and, what is more, non-convex. This means that the numerical solution may suffer from the hardware limitations and may not correspond to the global optimum.

Remark 5. The receding horizon technique (33) uses a discrete-time optimal control sequence parameterized by the discrete counterpart of the reference trajectory (20). As such, the usual performance, stability and robustness properties of the predictive control can be evoked only for the discrete time closed-loop behavior. The approach presented in this paper does not consider the intersample phenomena which can be handled using polytopic inclusions or alternative over-approximations (see Gielen et al., 2010; Heemels et al., 2010).

\section{Simulations}

This section proposes two simulation examples in order to better illustrate the proposed techniques.

Consider a set of $N_{a}$ heterogeneous agents in two spatial dimensions with the dynamics described by

$$
\begin{aligned}
A_{i} & =\left[\begin{array}{cccc}
0 & 0 & 1 & 0 \\
0 & 0 & 0 & 1 \\
0 & 0 & -\frac{\nu_{i}}{m_{i}} & 0 \\
0 & 0 & 0 & -\frac{\nu_{i}}{m_{i}}
\end{array}\right], \\
B_{i} & =\left[\begin{array}{cc}
0 & 0 \\
0 & 0 \\
\frac{1}{m_{i}} & 0 \\
0 & \frac{1}{m_{i}}
\end{array}\right]
\end{aligned}
$$

where $\left[\begin{array}{llll}x^{i} & y^{i} & v_{x}^{i} & v_{y}^{i}\end{array}\right]^{T},\left[\begin{array}{ll}u_{x}^{i} & u_{y}^{i}\end{array}\right]^{T}$ are the state and the input of each system. The components of the state are the position $\left(x^{i}, y^{i}\right)$ and the velocity $\left(v_{x}^{i}, v_{y}^{i}\right)$ of the $i$-th agent, $i=1, \ldots, N_{a}$. The parameters $m_{i}, \nu_{i}$ are the mass of Agent $i$ and the damping factor, respectively 5 .

In the first example, we consider a pointwise agent operating in an environment with obstacles designed as convex regions. Then, for controlling the agent to

\footnotetext{
${ }^{5}$ Note that the matrices $A_{i}$ and $B_{i}$ correspond to the continuous system 18 . After the initial stage of computing a flat trajectory, we will use discretized matrices, obtained as in Subsection 3.1 with a discretization time $T_{s}=0.1 \mathrm{~s}$
} 
maneuver successfully in the hostile environment we use first a gradient approach. Second, we introduce the potential function in a predictive control optimization problem such that the collision avoidance is satisfied. In the second example, we illustrate the trajectory tracking of a leader/followers formation. For avoiding collisions inside the formation, we consider agents with associated safety regions designed also as convex sets.

Example 1. Consider one agent (i.e., $N_{a}=1$ in (37) ) in two spatial dimensions described by the dynamics (37), with $m_{1}=45 \mathrm{~kg}, \nu_{1}=15 \mathrm{Ns} / \mathrm{m}$. Let the position component of the agent be constrained by three obstacles defined by (1). We consider a potential function as in (28) $\left(\beta_{r}=1, \beta_{a}=1\right)$ with two components: a repulsive potential (17) with $c_{3}=1, c_{4}=0.25$ and an attractive potential (32). The potential function generates a potential field depicted in Fig. 6. First, we calculate the gradient of the potential function which is piecewise affine as in 15. The negative value of the gradient is applied in order to derive a control action for the agent. We obtain that the obstacles are usually avoided, but there are situations when the constraints are not satisfied, or the control action obtained through the negative gradient has unrealistic values. For these reasons, we introduce the potential function in a predictive control framework as in (33), with a prediction horizon $N_{f}=2$. We obtain that the obstacles are always avoided. Figure 7 illustrates several trajectories of the agent with a random initial position.

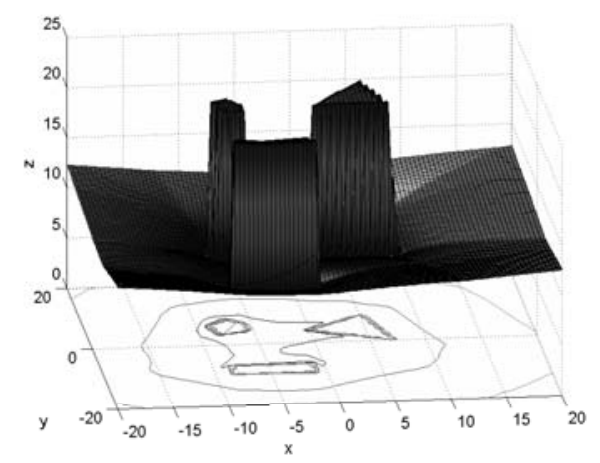

Fig. 6. Potential field in a workspace with three obstacles.

Example 2. Consider five agents (i.e., $N_{a}=5$ in (37) described by the dynamics (37), with $m_{1}=45 \mathrm{~kg}$, $m_{2}=60 \mathrm{~kg}, m_{3}=30 \mathrm{~kg}, m_{4}=50 \mathrm{~kg}, m_{5}=75$ $\mathrm{kg}, \nu_{1}=15 \mathrm{Ns} / \mathrm{m}, \nu_{2}=20 \mathrm{Ns} / \mathrm{m}, \nu_{3}=18 \mathrm{Ns} / \mathrm{m}$, $\nu_{4}=35 \mathrm{Ns} / \mathrm{m}, \nu_{5}=23 \mathrm{Ns} / \mathrm{m}$. The initial positions and velocities of the agents are chosen randomly. We associate with each agent a polyhedral safety region as in (1). For the sake of illustration, we shall choose identical safety zones for each agent. We take arbitrarily $l=1$ to be the

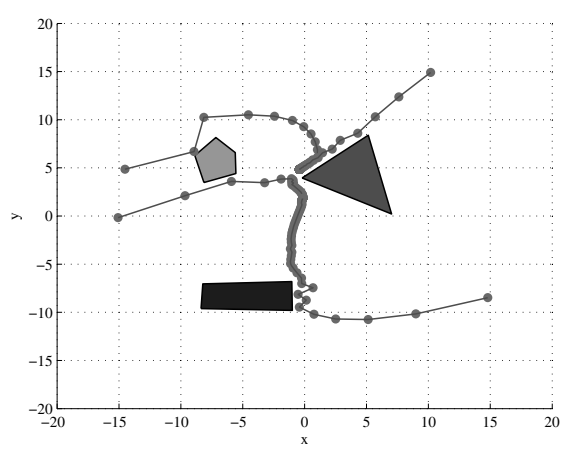

Fig. 7. Agent trajectories using predictive control.

leader which has to be followed by the rest of the agents $i=2, \ldots, 5(i \neq l)$. Figure 8 illustrates the potential field generated for the group of agents considered.

For the leader we generate through flatness methods, state and input references (20), and for both types of agents we use MPC in order to construct the control action. A quadratic cost function as defined in (24) is used for the leader. Figure 9 illustrates the reference trajectory and the time evolution of the leader along the trajectory. Satisfactory tracking performances for the given reference trajectory are obtained with a prediction horizon $N_{l}=10$.

For the followers we consider a potential function as the cost function in the optimization problem (33), with a prediction horizon $N_{f}=2$. The potential will be constructed such that both the following of the leader and the maintaining of a formation are respected. The neighborhood radius is set to $r=8 \mathrm{~m}$, the weighting coefficients are $\beta_{r}=1, \beta_{a}=10, c_{3}=1, c_{4}=0.25$, $\beta_{v}=15$. The effectiveness of the present algorithm is confirmed by the simulation depicted in Fig. 9 , where the evolution of the agents is represented at three different time instances. The agents successfully reach a formation and follow the leader without trespassing each other's safety regions.

We note that we prefer a smaller prediction horizon for the followers than the one used for the leader. This is justified by the fact that the trajectory of the leader is more important and that any additional prediction step for the potential function (which is not quadratic) incurs significant computational complexity.

Example 3. We build upon the previous example and we consider additionally obstacle avoidance. Furthermore, we redesign the reference trajectory such that it avoids stationary and a priori known obstacles. More precisely, we add control points which steer the reference trajectory from the interdicted region (for further details, see footnote 3 and the results of Prodan et al. (2012)).

In Fig. 10 the original reference illustrates the flat trajectory which does not take into account the obstacle. 
On the other hand, by adding an additional control point, we were able to construct a trajectory which avoids the obstacle. Satisfactory tracking performances for the given reference trajectory are obtained with a prediction horizon $N_{l}=10$, as well as in the previous example.

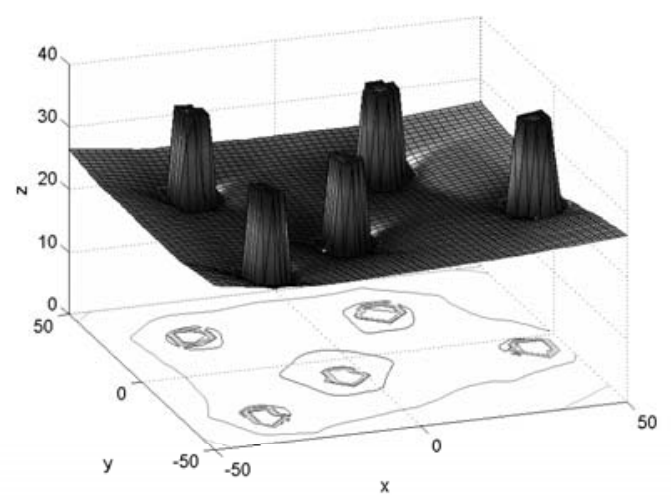

Fig. 8. Potential field in a workspace with five agents.

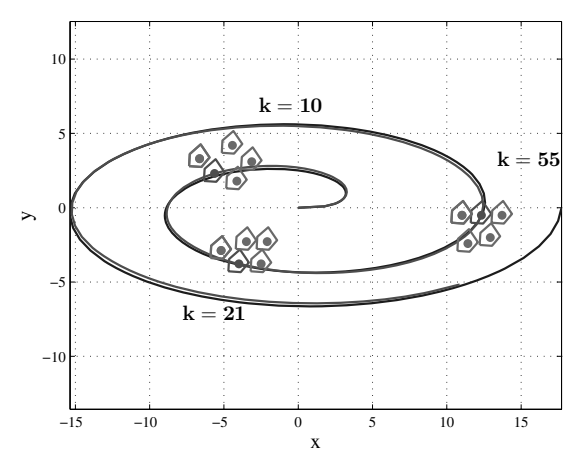

Fig. 9. Trajectory tracking of the leader/followers formation at different time instances with their safety regions.

\section{Conclusions}

This paper presents the trajectory tracking problem of multiple agents. Convex safety regions are associated with each agent in order to solve the collision avoidance problem. First, the notion of a polyhedral function is recalled and further introduced in a potential function which accounts for the associated safety region. Second, in real-time, a receding horizon control design and a leader/followers strategy are adopted for driving the agents into a formation with a collision free behavior. For the leader, a flat trajectory is generated and a receding horizon optimization problem is solved in order to minimize the tracking error. For the followers, a decentralized control method is introduced by combining

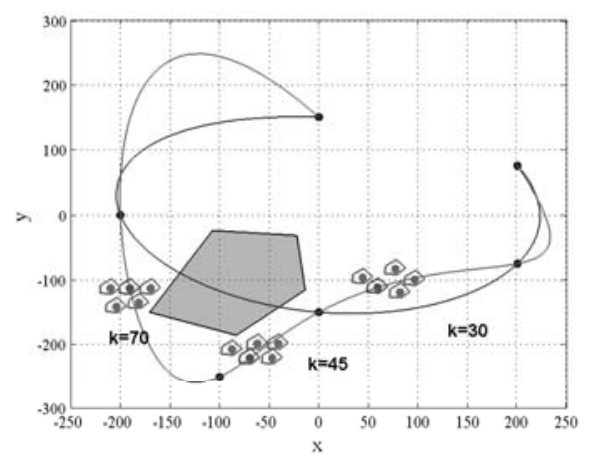

Fig. 10. Obstacle avoidance and trajectory tracking of the leader/followers formation at different time instances with their safety regions.

the model predictive control and potential field concepts. Two kinds of potential terms are distinguished in the cost function of the followers. The repulsive potential term accounts for collision avoidance between the agents, and the attractive potential term guarantees the convergence to a formation and the following of the leader.

Future work will focus on the investigation of the robust stability properties of the multi-agent system in presence of disturbances and uncertainties, problem being known to be particularly intricate without strict assumptions on the time-varying properties of the interconnection graph.

\section{Acknowledgment}

The research of Ionela Prodan is financially supported by the EADS Corporate Foundation (091-A009-1006).

\section{References}

Baskar, L., De Schutter, B. and Hellendoorn, H. (2006) Decentralized traffic control and management with intelligent vehicles, Proceedings of the 9th TRAIL Congress, Delft, The Netherlands, (CD-ROM).

Bemporad, A. and Morari, M. (1999). Control of systems integrating logic, dynamics, and constraints, Automatica 35(3): 407-427.

Blanchini, F. (1995). Nonquadratic Lyapunov functions for robust control, Automatica 31(3): 451-461.

Camacho, E. and Bordons, C. (2004). Model Predictive Control, Springer-Verlag, London.

De Doná, J., Suryawan, F., Seron, M. and Lévine, J. (2009). A flatness-based iterative method for reference trajectory generation in constrained NMPC, in L. Magni, D.M. Raimondo and F. Allgöwer (Eds.), Nonlinear Model Predictive Control, Springer-Verlag, pp. 325-333.

Dunbar, W. and Murray, R. (2006). Distributed receding horizon control for multi-vehicle formation stabilization, Automatica 42(4): 549-558. 
Fang, Z., Song, W., Zhang, J. and Wu, H. (2010). Experiment and modeling of exit-selecting behaviors during a building evacuation, Statistical Mechanics and Its Applications 389(4): 815-824.

Fliess, M., Lévine, J., Martin, P. and Rouchon, P. (1995). Flatness and defect of non-linear systems: Introductory theory and examples, International Journal of Control 61(6): 1327-1361.

Garey, M. and Johnson, D. (1979). Computers and Intractability. A Guide to the Theory of NP-completeness, WH Freeman and Company, San Francisco, CA.

Gielen, R., Olaru, S., Lazar, M., Heemels, W., Van de Wouw, N. and Niculescu, S. (2010). On polytopic inclusions as a modeling framework for systems with time-varying delays, Automatica 46(3): 615-619.

Girard, A., de Sousa, J. and Hedrick, J. (2001). Coordinated control of agent formations in uncertain, dynamic environments, Proceedings of the European Control Conference, Porto, Portugal, pp. 121-127.

Goodwin, G., Seron, M., Middleton, R., Zhang, M., Hennessy, B., Stone, P. and Menabde, M. (2006). Receding horizon control applied to optimal mine planning, Automatica 42(8): 1337-1342.

Heemels, W., Van De Wouw, N., Gielen, R., Donkers, M., Hetel, L., Olaru, S., Lazar, M., Daafouz, J. and Niculescu, S. (2010). Comparison of overapproximation methods for stability analysis of networked control systems, Proceedings of the 13th ACM International Conference on Hybrid Systems: Computation and Control, Stockholm, Sweden, pp. 181-190.

Helbing, D., Farkas, I. and Vicsek, T. (2000). Simulating dynamical features of escape panic, Nature 407(6803): 487-490.

Jadbabaie, A., Lin, J. and Morse, A. (2003). Coordination of groups of mobile autonomous agents using nearest neighbor rules, IEEE Transactions on Automatic Control 48(6): 988-1001.

Khatib, O. (1986). Real-time obstacle avoidance for manipulators and mobile robots, International Journal of Robotics Research 5(1): 90.

Maciejowski, J. (2002). Predictive Control with Constraints, Pearson Education, Harlow.

Massion, I., Keviczky, T. and Verhaegen, M. (2008). New approaches to distributed control of satellite formation flying, Proceedings of the 3rd International Symposium on Formation Flying, Missions and Technologies, Noordwijk, The Netherlands, pp. 22-27.

Mayne, D., Rawlings, J., Rao, C. and Scokaert, P. O. (2000). Constrained model predictive control: Stability and optimality, Automatica 36(6): 789-814.

Mazur, A. and Szakiel, D. (2009). On path following control of nonholonomic mobile manipulators, International Journal of Applied Mathematics and Computer Science 19(4): 561-574, DOI: 10.2478/v10006-009-0044-0.
Mesbahi, M. and Hadaegh, F. (2001). Formation flying control of multiple spacecraft via graphs, matrix inequalities, and switching, Journal of Guidance, Control, and Dynamics 24(2): 369-377.

Michałek, M., Dutkiewicz, P., Kiełczewski, M. and Pazderski, D. (2009). Trajectory tracking for a mobile robot with skid-slip compensation in the vector-field-orientation control system, International Journal of Applied Mathematics and Computer Science 19(4): 547-559, DOI: 10.2478/v10006-009-0043-1.

Negenborn, R., van Overloop, P., Keviczky, T. and De Schutter, B. (2009). Distributed model predictive control of irrigation canals, Networks and Heterogeneous Media 4(2): 359-380.

Osiadacz, A., Nemhauser, G.L. and Wolsey, L.A. (1990). Integer and combinatorial optimization, International Journal of Adaptive Control and Signal Processing 4(4): 333-334.

Overloop, P., Negenborn, R., Schutter, B. and Giesen, N. (2010). Predictive control for national water flow optimization in the Netherlands, in R.R. Negenborn, Z. Lukszo and H. Hellendoorn (Eds.), Intelligent Infrastructures, Springer, pp. 439-461.

Prodan, I., Bencatel, R., Olaru, S., Sousa, J., Stoica, C. and Niculescu, S. (2012). Predictive control for autonomous aerial vehicles trajectory tracking, Proceedings of the Nonlinear Model Predictive Control Conference, Noordwijkerhout, The Netherlands, pp. 508-513.

Prodan, I., Olaru, S., Stoica, C. and Niculescu, S. (2010). Collision avoidance and path following for multi-agent dynamical systems, 2010 International Conference on Control Automation and Systems (ICCAS), Gyeonggi-do, Seoul, Korea, pp. 1930-1935.

Prodan, I., Olaru, S., Stoica, C. and Niculescu, S.-I. (2011). Predictive control for tight group formation of multi-agent systems, Proceedings of the 18th IFAC World Congress, Milano, Italy, pp. 138-143.

Richards, A. and How, J. (2002). Aircraft trajectory planning with collision avoidance using mixed integer linear programming, Proceedings of the 21st American Control Conference, Anchorage, AL, USA, pp. 1936-1941.

Rimon, E. and Koditschek, D. (1992). Exact robot navigation using artificial potential functions, IEEE Transactions on Robotics and Automation 8(5): 501-518.

Rossiter, J. (2003). Model-Based Predictive Control: A Practical Approach, CRC Press, Boca Raton, FL.

Roussos, G. and Kyriakopoulos, K. (2010). Completely decentralised navigation of multiple unicycle agents with prioritization and fault tolerance, Proceedings of the 49th IEEE Conference on Decision and Control, Atlanta, GA, USA, pp. 1372-1377.

Stoican, F., Prodan, I. and Olaru, S. (2011). On the hyperplanes arrangements in mixed-integer techniques, Proceedings of the 30th American Control Conference, San Francisco, CA, USA, pp. 1898-1903. 
Suryawan, F., De Dona, J. and Seron, M. (2010). Methods for trajectory generation in a magnetic-levitation system under constraints, 18th Mediterranean Conference on Control and Automation, Marrakech, Morocco, pp. 945-950.

Tanner, H., Jadbabaie, A. and Pappas, G. (2007). Flocking in fixed and switching networks, IEEE Transactions on Automatic Control 52(5): 863-868.

Van den Berg, M., Hegyi, A., De Schutter, B. and Hellendoorn, J. (2004). A macroscopic traffic flow model for integrated control of freeway and urban traffic networks, Proceedings of the 43rd IEEE Conference on Decision and Control, Atlantis, Paradise Island, The Bahamas, Vol. 3, pp. 2774-2779.

Van Nieuwstadt, M. and Murray, R. (1998). Real-time trajectory generation for differentially flat systems, International Journal of Robust and Nonlinear Control 8(11): 995-1020.

Ziegler, G.M. (1995). Lectures on Polytopes, Springer-Verlag, New York, NY.

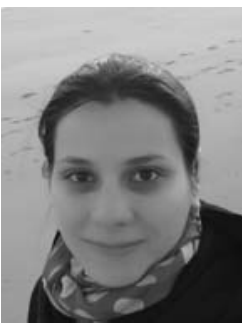

Ionela Prodan received the B.Eng. degree from University "Politehnica" Bucharest, Romania, in 2009 and the Ph.D. degree in automatic control from SUPELEC, France. Her research interests include control of multi-agent dynamical systems via model predictive control, set-theoretic methods and mixed-integer programming, as well as applications for trajectory tracking and formation control of Unmanned Aerial Vehicles (UAVs)

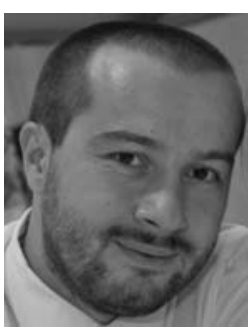

Sorin Olaru received the M.Sc. degree from University "Politehnica" Bucharest, Romania, and the Ph.D. degree from University Paris XI, France. Since 2001 he has held various positions at INRIA and SUPELEC in France as well as visiting appointments at the University of Newcastle, Australia, and NTNU Trondheim, Norway. Currently he is a professor in SUPELEC and a member of the INRIA Disco team. His research interests include optimization-based control design and set theoretic characterization of constrained dynamical systems.

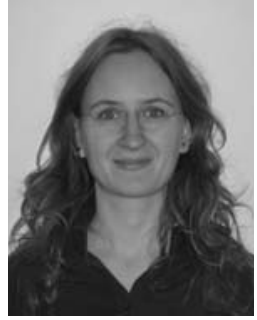

Cristina Stoica received the B.Sc. (2004) and the M.Sc. (2005) degrees in electrical engineering from University "Politehnica" Bucharest, and the Ph.D. degree (2008) in automatic control from University Paris XI, France. She is currently an assistant professor (since 2008) at SUPELEC Systems Sciences (E3S), Automatic Control Department. Her main research activities include predictive control, robust control, and uncertain systems.

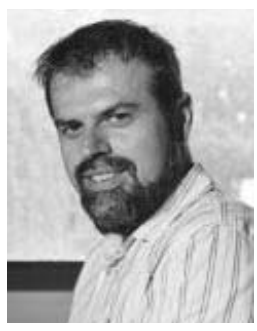

Silviu-Iulian Niculescu received the B.Sc. degree from the Polytechnical Institute of Bucharest, Romania, and the M.Sc. and Ph.D. degrees, in automatic control, from INP Grenoble, France. From 1992 to 1997, he was with the Department of Automatic Control and Computers, University "Politehnica" Bucharest, Romania. From 1997 to 2006, he was with the HEUDIASYC (heuristics and diagnosis of complex systems) laboratory, Compiègne, France, as a researcher at CNRS (French National Center for Scientific Research). He also held a postdoctoral position in the Department of Applied Mathematics, ENSTA, Paris, France, from 1996 to 1997. In September 2006, he joined L2S (Laboratory of Signals and Systems), Gif-sur-Yvette, where he is currently a research director (senior researcher) at CNRS and the head of the laboratory. His research interests include delay systems, robust control, operator theory, and numerical methods in optimization and their applications to the design of engineering systems.

Received: 30 October 2011 Revised: 29 May 2012 